\title{
Contact Riemannian Manifolds with Constant $\varphi$-Sectional Curvature
}

\author{
Themis KOUFOGIORGOS* \\ University of Ioannina \\ (Communicated by N. Sasakura)
}

\begin{abstract}
In this paper a class of contact, non Sasakian, Riemannian manifolds of constant $\varphi$-sectional curvature is found and studied.
\end{abstract}

\section{Introduction.}

Let $M$ be a Riemannian manifold. It is well known [1, p. 131] that the tangent sphere bundle $T_{1} M$ admits a contact Riemannian structure $(\eta, \xi, \varphi, g) . T_{1} M$ together with this structure is a contact Riemannian manifold. If $M$ is of constant sectional curvature $c=1$, then $T_{1} M$ is a Sasakian manifold [9], i.e. its curvature tensor $R$ satisfies $R(X, Y) \xi=\eta(Y) X-\eta(X) Y$ for all vector fields $X, Y$. If $c=0$, then the curvature tensor of $T_{1} M$ satisfies the condition $R(X, Y) \xi=0$ [2]. Applying a $D$-homothetic deformation on a contact Riemannian manifold satisfying $R(X, Y) \xi=0$, we get a contact Riemannian manifold such that $R(X, Y) \xi=\kappa(\eta(Y) X-\eta(X) Y)+\mu(\eta(Y) h X-\eta(X) h Y)$, where $\kappa, \mu$ are real constants and $2 h$ is the Lie differentiation of $\varphi$ in the direction of $\xi$. We call this kind of manifold $(\kappa, \mu)$-contact Riemannian manifold. The above construction was done in [5] and the study of $(\kappa, \mu)$-contact Riemannian manifolds has begun in [3]. Examples of such manifolds exist in all dimensions. The 3-dimensional non Sasakian, $(\kappa, \mu)-$ contact Riemannian manifolds have constant $\varphi$-sectional curvature, but for higher dimensions this is not, in general, true.

Our purpose in this paper is to find conditions, which characterize $(\kappa, \mu)$-contact Riemannian manifolds with constant $\varphi$-sectional curvature. At first we prove that if the $\varphi$-sectional curvature at a point $P$ of a $(2 n+1)$-dimensional $(\kappa, \mu)$-contact Riemannian manifold $M(n>1)$ is independent of the $\varphi$-section at $P$, then it is constant. This result is analogous to Schur's theorem and extends a corresponding result, which is valid on Sasakian manifolds. Our second result states that a non Sasakian, $(\kappa, \mu)$ contact Riemannian manifold is of constant $\varphi$-sectional curvature if and only if $\mu=$

*Research supported by E.E.C. contract CHRX-CT94-0661 
$\kappa+1$. Therefore in this case an explicit expression for the curvature tensor is given. As an application of the last statement we prove that the tangent sphere bundle, of a Riemannian manifold of constant sectional curvature $c$, is of constant $\varphi$-sectional curvature iff $c=2 \pm \sqrt{5}$. Finally we give a method to construct $(\kappa, \mu)$-contact Riemannian manifolds of constant $\varphi$-sectional curvature. It seems that these manifolds are the first examples of non Sasakian, contact Riemannian manifolds with constant $\varphi$ sectional curvature.

\section{Contact Riemannian manifolds.}

A differential 1-form $\eta$ on a differentiable $(2 n+1)$-dimensional manifold $M$ is called a contact form if it satisfies $\eta \wedge(d \eta)^{n} \neq 0$ everywhere on $M$. By a contact manifold $(M, \eta)$ we mean a manifold $M$ together with a contact form $\eta$. Since $d \eta$ is of rank $2 n$, there exists a global vector field $\xi$, called the characteristic vector field, such that $\eta(\xi)=1$ and $\mathscr{L}_{\xi} \eta=0$, where $\mathscr{L}_{\xi}$ denotes the Lie differentiation by $\xi$. Moreover it is well known that there exist a Riemannian metric $g$ and a $(1,1)$-tensor field $\varphi$ satisfying

$$
\begin{gathered}
\varphi \xi=0, \quad \eta \circ \varphi=0, \quad g(X, \xi)=\eta(X) \\
\varphi^{2}=-I+\eta \otimes \xi, \quad d \eta(X, Y)=g(X, \varphi Y) \\
g(\varphi X, \varphi Y)=g(X, Y)-\eta(X) \eta(Y)
\end{gathered}
$$

for all vector fields $X, Y$ on $M$. The structure $(\eta, \xi, \varphi, g)$ is called a contact Riemannian structure and the manifold $M$ carrying such a structure is said to be a contact Riemannian manifold.

Following [1], we define the $(1,1)$-type tensor field $h$ by $2 h=\mathscr{L}_{\xi} \varphi$. Then $h$ satisfies the relations

$$
h \xi=0, \quad \operatorname{Tr} h=\operatorname{Tr} h \varphi=0, \quad h \varphi+\varphi h=0 .
$$

The contact form $\eta$ on $M$ gives rise to an almost complex structure on the product $M \times R$. If this structure is integrable, then the contact Riemannian manifold is said to be Sasakian. Equivalently, a contact Riemannian manifold is Sasakian if and only if $R(X, Y) \xi=\eta(Y) X-\eta(X) Y$ for all vector fields $X, Y$. The sectional curvature $K(X, \varphi X)$ of a plane section spanned by a vector $X$ orthogonal to $\xi$ is called a $\varphi$-sectional curvature.

The tangent sphere bundle $T_{1} M$ of a Riemannian manifold $M$ admits a contact Riemannian structure, known as the standard contact Riemannian structure. From now on, when we refer to $T_{1} M$ we will consider it equipped with the standard contact Riemannian structure.

For more details concerning contact Riemannian manifolds and related topics we refer the reader to [1]. 


\section{3. $(\kappa, \mu)$-contact Riemannian manifolds.}

For real constants $\kappa, \mu$, the $(\kappa, \mu)$-nullity distribution of a contact Riemannian manifold $M(\eta, \xi, \varphi, g)$ is a distribution

$$
\begin{aligned}
N(\kappa, \mu): P \rightarrow N_{P}(\kappa, \mu)=\left\{Z \in T_{P} M \mid R(X, Y) Z\right. & =\kappa(g(Y, Z) X-g(X, Z) Y) \\
& +\mu(g(Y, Z) h X-g(X, Z) h Y)\}
\end{aligned}
$$

where $R$ is the curvature tensor of $M$. So, if the characteristic vector field $\xi$ belongs to the $(\kappa, \mu)$-nullity distribution we have

$$
R(X, Y) \xi=\kappa(\eta(Y) X-\eta(X) Y)+\mu(\eta(Y) h X-\eta(X) h Y) .
$$

We call $(\kappa, \mu)$-contact Riemannian manifold, a contact Riemannian manifold satisfying (3.1). The class of $(\kappa, \mu)$-contact Riemannian manifolds contains the class of Sasakian manifolds, which we get for $\kappa=1$ (and hence $h=0$, by (3.2)). Characteristic examples of non Sasakian, $(\kappa, \mu)$-contact Riemannian manifolds are the tangent sphere bundles of Riemannian manifolds of constant sectional curvature $c \neq 1$. Especially in the 3dimensional case this class contains the Lie groups $S U(2), S O(3), S L(2, R), O(1,2), E(2)$, $E(1,1)$ with a left invariant metric. For more examples see [3].

From now on, we suppose $M(\eta, \xi, \varphi, g)$ is a $(2 n+1)$-dimensional $(\kappa, \mu)$-contact Riemannian manifold. In [3] the following formulas have been proved:

$$
h^{2}=(\kappa-1) \varphi^{2}
$$

(so $\kappa \leq 1$ and $\kappa=1$ iff $M$ is a Sasakian manifold),

$$
\begin{gathered}
R(\xi, X) Y=\kappa(g(X, Y) \xi-\eta(Y) X)+\mu(g(h X, Y) \xi-\eta(Y) h X) \\
R(X, Y) \varphi Z=\varphi R(X, Y) Z+\{(1-\kappa)[\eta(X) g(\varphi Y, Z)-\eta(Y) g(\varphi X, Z)] \\
+(1-\mu)[\eta(X) g(\varphi h Y, Z)-\eta(Y) g(\varphi h X, Z)]\} \xi \\
-g(Y+h Y, Z)(\varphi X+\varphi h X)+g(X+h X, Z)(\varphi Y+\varphi h Y) \\
-g(\varphi Y+\varphi h Y, Z)(X+h X)+g(\varphi X+\varphi h X, Z)(Y+h Y) \\
-\eta(Z)\{(1-\kappa)[\eta(X) \varphi Y-\eta(Y) \varphi X]+(1-\mu)[\eta(X) \varphi h Y-\eta(Y) \varphi h X]\} .
\end{gathered}
$$

Moreover in [3] the following results have been proved.

LEMMA 3.1. If $\kappa<1$, then $M$ admits three mutually orthogonal and integrable distributions $D(0), D(\lambda), D(-\lambda)$, defined by the eigenspaces of $h$, where $\lambda=\sqrt{1-\kappa}$.

THEOREM 3.2. If $\kappa<1$, then

$$
\begin{gathered}
R\left(X_{\lambda}, Y_{\lambda}\right) Z_{-\lambda}=(\kappa-\mu)\left[g\left(\varphi Y_{\lambda}, Z_{-\lambda}\right) \varphi X_{\lambda}-g\left(\varphi X_{\lambda}, Z_{-\lambda}\right) \varphi Y_{\lambda}\right] \\
R\left(X_{-\lambda}, Y_{-\lambda}\right) Z_{\lambda}=(\kappa-\mu)\left[g\left(\varphi Y_{-\lambda}, Z_{\lambda}\right) \varphi X_{-\lambda}-g\left(\varphi X_{-\lambda}, Z_{\lambda}\right) \varphi Y_{-\lambda}\right] \\
R\left(X_{\lambda}, Y_{-\lambda}\right) Z_{-\lambda}=\kappa g\left(\varphi X_{\lambda}, Z_{-\lambda}\right) \varphi Y_{-\lambda}+\mu g\left(\varphi X_{\lambda}, Y_{-\lambda}\right) \varphi Z_{-\lambda}
\end{gathered}
$$




$$
\begin{gathered}
R\left(X_{\lambda}, Y_{-\lambda}\right) Z_{\lambda}=-\kappa g\left(\varphi Y_{-\lambda}, Z_{\lambda}\right) \varphi X_{\lambda}-\mu g\left(\varphi Y_{-\lambda}, X_{\lambda}\right) \varphi Z_{\lambda} \\
R\left(X_{\lambda}, Y_{\lambda}\right) Z_{\lambda}=[2(1+\lambda)-\mu]\left[g\left(Y_{\lambda}, Z_{\lambda}\right) X_{\lambda}-g\left(X_{\lambda}, Z_{\lambda}\right) Y_{\lambda}\right] \\
R\left(X_{-\lambda}, Y_{-\lambda}\right) Z_{-\lambda}=[2(1-\lambda)-\mu]\left[g\left(Y_{-\lambda}, Z_{-\lambda}\right) X_{-\lambda}-g\left(X_{-\lambda}, Z_{-\lambda}\right) Y_{-\lambda}\right]
\end{gathered}
$$

where $X_{\lambda}, Y_{\lambda}, Z_{\lambda}$ and $X_{-\lambda}, Y_{-\lambda}, Z_{-\lambda}$ are the components of $X, Y, Z$ on $D(\lambda)$ and $D(-\lambda)$ respectively.

THEOREM 3.3. If $\kappa<1$, then

1) The sectional curvature of a plane section $(X, Y)$ orthogonal to $\xi$ with $X \in D(\lambda)$ and $Y \in D(-\lambda)$ is given by

$$
K(X, Y)=-(\kappa+\mu) g(X, \varphi Y)^{2} .
$$

2) The Ricci operator is given by

$$
Q=(2(n-1)-n \mu) I+(2(n-1)+\mu) h+(2(1-n)+n(2 \kappa+\mu)) \eta \otimes \xi .
$$

THEOREM 3.4. The tangent sphere bundle $T_{1} M$ is $a(\kappa, \mu)$-contact Riemannian manifold if and only if the base manifold $M$ is of constant sectional curvature $c$. Moreover $\kappa=c(2-c)$ and $\mu=-2 c$.

\section{Main results.}

Let $M(\eta, \xi, \varphi, g)$ be a $(2 n+1)$-dimensional $(\kappa, \mu)$-contact Riemannian manifold. If $n=1$, and $\kappa \neq 1$, then it is well known [3] that $M$ is of constant $\varphi$-sectional curvature. In the next theorem we consider the case $n>1$ and we give a necessary condition so that $M$ is of constant $\varphi$-sectional curvature. This theorem extends two theorems of Ogiue (see [6] or [1]) and Endo [4], which are valid for $\kappa=1$ and $\mu=0$ respectively.

THEOREM 4.1. Let $M(\eta, \xi, \varphi, g)$ be a $(2 n+1)$-dimensional $(\kappa, \mu)$-contact Riemannian manifold $(n>1)$. If the $\varphi$-sectional curvature of any point of $M$ is independent of the choice of $\varphi$-section at the point, then it is constant on $M$ and the curvature tensor is given by

$$
\begin{gathered}
4 R(X, Y) Z=(H+3)\{g(Y, Z) X-g(X, Z) Y\}+(H+3-4 \kappa)\{\eta(X) \eta(Z) Y \\
-\eta(Y) \eta(Z) X+g(X, Z) \eta(Y) \xi-g(Y, Z) \eta(X) \xi\} \\
+(H-1)\{2 g(X, \varphi Y) \varphi Z+g(X, \varphi Z) \varphi Y-g(Y, \varphi Z) \varphi X\} \\
-2\{g(h X, Z) h Y-g(h Y, Z) h X+2 g(X, Z) h Y-2 g(Y, Z) h X-2 \eta(X) \eta(Z) h Y \\
+2 \eta(Y) \eta(Z) h X+2 g(h X, Z) Y-2 g(h Y, Z) X+2 g(h Y, Z) \eta(X) \xi \\
-2 g(h X, Z) \eta(Y) \xi-g(\varphi h X, Z) \varphi h Y+g(\varphi h Y, Z) \varphi h X\} \\
+4 \mu\{\eta(Y) \eta(Z) h X-\eta(X) \eta(Z) h Y+g(h Y, Z) \eta(X) \xi-g(h X, Z) \eta(Y) \xi\}
\end{gathered}
$$


where $H$ is the constant $\varphi$-sectional curvature. Moreover if $\kappa \neq 1$, then $\mu=\kappa+1$ and $H=-2 \kappa-1$.

Proof. For the Sasakian case $\kappa=1$, the proof is known ([1], p. 97). So we have to prove the theorem for $\kappa \neq 1$. Let $P \in M$ and $X, Y \in T_{P} M$ orthogonal to $\xi$. Using the first identity of Bianchi, the basic properties of the curvature tensor, $\varphi$ is antisymmetric, $h$ is symmetric, (2.2) and (2.3) we get from (3.4), successively:

$$
\begin{gathered}
g(R(X, \varphi X) Y, \varphi Y)=g(R(X, \varphi Y) Y, \varphi X)+g(R(X, Y) X, Y) \\
-g(X, Y)^{2}-g(h X, Y)^{2}-2 g(X, Y) g(h X, Y)+g(X, X) g(Y, Y) \\
+g(X, X) g(h Y, Y)+g(Y, Y) g(h X, X)+g(h X, X) g(h Y, Y) \\
-g(\varphi X, Y)^{2}+g(\varphi h X, Y)^{2}-g(\varphi h X, X) g(\varphi h Y, Y) \\
g(R(X, \varphi Y) X, \varphi Y)=g(R(X, \varphi Y) Y, \varphi X) \\
+g(X, Y)^{2}-g(h X, Y)^{2}-g(\varphi h X, X) g(\varphi h Y, Y)-g(X, X) g(Y, Y) \\
-g(Y, Y) g(h X, X)+g(X, X) g(h Y, Y)+g(h X, X) g(h Y, Y) \\
+g(\varphi X, Y)^{2}+g(\varphi h X, Y)^{2}+2 g(\varphi X, Y) g(\varphi h X, Y) \\
g(R(Y, \varphi X) Y, \varphi X)=g(R(X, \varphi Y) Y, \varphi X) \\
+g(X, Y)^{2}-g(h X, Y)^{2}-g(\varphi h X, X) g(\varphi h Y, Y)+g(\varphi X, Y)^{2} \\
+g(\varphi h X, Y)^{2}-2 g(\varphi X, Y) g(\varphi h X, Y)-g(X, X) g(Y, Y) \\
-g(X, X) g(h Y, Y)+g(Y, Y) g(h X, X)+g(h X, X) g(h Y, Y) \\
g(R(X, Y) \varphi X, \varphi Y)=g(R(X, Y) X, Y) \\
-g(X, Y)^{2}-g(h X, Y)^{2}-2 g(X, Y) g(h X, Y)+g(X, X) g(Y, Y) \\
+g(X, X) g(h Y, Y)+g(Y, Y) g(h X, X)+g(h X, X) g(h Y, Y) \\
-g(\varphi X, Y)^{2}+g(\varphi h X, Y)^{2}-g(\varphi h X, X) g(\varphi h Y, Y) .
\end{gathered}
$$

We now suppose that the $\varphi$-sectional curvature at $P$ is independent of the $\varphi$-section at $P$, i.e. $K(X, \varphi X)=H(P)$ for any $X \in T_{P} M$ orthogonal to $\xi$. Let $X, Y \in T_{P} M$ and $X, Y$ orthogonal to $\xi$. From

$$
\begin{aligned}
& g(R(X+Y, \varphi X+\varphi Y)(X+Y), \varphi X+\varphi Y)=-H(P) g(X+Y, X+Y)^{2} \\
& g(R(X-Y, \varphi X-\varphi Y)(X-Y), \varphi X-\varphi Y)=-H(P) g(X-Y, X-Y)^{2}
\end{aligned}
$$

we get by a straightforward calculation

$$
\begin{gathered}
2 g(R(X, \varphi X) Y, \varphi Y)+ \\
=(R(X, \varphi Y) X, \varphi Y)+2 g(R(X, \varphi Y) Y, \varphi X)+g(R(Y, \varphi X) Y, \varphi X) \\
=-2 H(P)\left\{2 g(X, Y)^{2}+g(X, X) g(Y, Y)\right\} .
\end{gathered}
$$

Combining (4.2), (4.3), (4.4) and (4.6) we get 


$$
\begin{gathered}
3 g(R(X, \varphi Y) Y, \varphi X)+g(R(X, Y) X, Y) \\
-2 g(h X, Y)^{2}-2 g(X, Y) g(h X, Y)+g(X, X) g(h Y, Y)+g(Y, Y) g(h X, X) \\
+2 g(h X, X) g(h Y, Y)+2 g(\varphi h X, Y)^{2}-2 g(\varphi h X, X) g(\varphi h Y, Y) \\
=-H(P)\left\{2 g(X, Y)^{2}+g(X, X) g(Y, Y)\right\} .
\end{gathered}
$$

Replacing $Y$ by $\varphi Y$ in (4.7) and using (2.3) and (2.4) we have

$$
-3 g(R(X, Y) \varphi Y, \varphi X)+g(R(X, \varphi Y) X, \varphi Y)
$$

$-2 g(\varphi h X, Y)^{2}+2 g(X, \varphi Y) g(\varphi h X, Y)-g(X, X) g(h Y, Y)+g(Y, Y) g(h X, X)$

$-2 g(h X, X) g(h Y, Y)+2 g(h X, Y)^{2}+2 g(\varphi h X, X) g(\varphi h Y, Y)$

$$
=-H(P)\left\{2 g(X, \varphi Y)^{2}+g(X, X) g(Y, Y)\right\} \text {. }
$$

Combining (4.8) with (4.3) and (4.5) we finally get

$$
\begin{gathered}
3 g(R(X, Y) X, Y)+g(R(X, \varphi Y) Y, \varphi X) \\
-2 g(X, Y)^{2}-2 g(h X, Y)^{2}-6 g(X, Y) g(h X, Y)+2 g(X, X) g(Y, Y) \\
+3 g(X, X) g(h Y, Y)+3 g(Y, Y) g(h X, X)+2 g(h X, X) g(h Y, Y) \\
-2 g(X, \varphi Y)^{2}+2 g(\varphi h X, Y)^{2}-2 g(\varphi h X, Y) g(\varphi h Y, Y) \\
=-H(P)\left\{2 g(X, \varphi Y)^{2}+g(X, X) g(Y, Y)\right\} .
\end{gathered}
$$

Now, (4.9) together with (4.7) yield

$$
\begin{gathered}
4 g(R(X, Y) Y, X)=(H(P)+3)\left\{g(X, X) g(Y, Y)-g(X, Y)^{2}\right\}+3(H(P)-1) g(X, \varphi Y)^{2} \\
-2\left\{g(h X, Y)^{2}+4 g(X, Y) g(h X, Y)-2 g(X, X) g(h Y, Y)-2 g(Y, Y) g(h X, X)\right. \\
\left.-g(h X, X) g(h Y, Y)-g(\varphi h X, Y)^{2}+g(\varphi h X, X) g(\varphi h Y, Y)\right\}
\end{gathered}
$$

for any $X, Y \in T_{P} M$ and $X, Y$ orthogonal to $\xi$. Let $X, Y, Z \in T_{P} M$ and $X, Y, Z$ orthogonal to $\xi$. Applying (4.10) in

$$
g(R(X+Z, Y) Y, X+Z)=g(R(X, Y) Y, X)+g(R(Z, Y) Y, Z)+g(R(X, Y) Y, Z)
$$

we finally get

$$
\begin{gathered}
4 g(R(X, Y) Y, Z)=(H(P)+3)\{g(X, Z) g(Y, Y)-g(X, Y) g(Y, Z)\} \\
+3(H(P)-1) g(X, \varphi Y) g(Z, \varphi Y)-2\{g(h X, Y) g(h Z, Y)+2 g(X, Y) g(h Z, Y) \\
+2 g(Z, Y) g(h X, Y)-2 g(X, Z) g(h Y, Y)-2 g(Y, Y) g(h X, Z) \\
-g(h X, Z) g(h Y, Y)-g(\varphi h X, Y) g(\varphi h Z, Y)+g(\varphi h X, Z) g(\varphi h Y, Y)\} .
\end{gathered}
$$

Moreover, using (3.1), (2.1) and $h \varphi$ is symmetric, it is easy to check that (4.11) is valid for any $Z$ and for $X, Y$ orthogonal to $\xi$. Hence (4.11) is reduced to

$$
\begin{gathered}
R(X, Y) Y=(H(P)+3)\{g(Y, Y) X-g(X, Y) Y\}+3(H(P)-1) g(X, \varphi Y) \varphi Y \\
-2\{g(h X, Y) h Y+2 g(X, Y) h Y+2 g(h X, Y) Y-2 g(h Y, Y) X
\end{gathered}
$$




$$
-2 g(Y, Y) h X-g(h Y, Y) h X-g(\varphi h X, Y) \varphi h Y+g(\varphi h Y, Y) \varphi h X\}
$$

for any $X, Y$ orthogonal to $\xi$. Now let $X, Y, Z$ be orthogonal to $\xi$. Replacing in (4.12) $Y$ by $Y+Z$ and using (4.12) we get

$$
\begin{aligned}
& 4\{R(X, Y) Z+R(X, Z) Y\}=(H(P)+3)\{2 g(Y, Z) X-g(X, Y) Z-g(X, Z) Y\} \\
& +3(H(P)-1)\{g(X, \varphi Y) \varphi Z+g(X, \varphi Z) \varphi Y\} \\
& -2\{g(h X, Y) h Z+g(h X, Z) h Y+2 g(X, Y) h Z+2 g(X, Z) h Y+2 g(h X, Y) Z \\
& +2 g(h X, Z) Y-4 g(h Y, Z) X-4 g(Y, Z) h X-2 g(h Y, Z) h X \\
& -g(\varphi h X, Y) \varphi h Z-g(\varphi h X, Z) \varphi h Y+2 g(\varphi h Y, Z) \varphi h X\} \text {. }
\end{aligned}
$$

Replacing $X$ by $Y$ and $Y$ by $-X$ in (4.13) we have

$$
\begin{gathered}
4\{R(X, Y) Z+R(Z, Y) X\}=(H(P)+3)\{-2 g(X, Z) Y+g(X, Y) Z+g(Y, Z) X\} \\
+3(H(P)-1)\{-g(\varphi X, Y) \varphi Z-g(\varphi Z, Y) \varphi X\} \\
-2\{-g(h Y, X) h Z-g(h Y, Z) h X-2 g(X, Y) h Z-2 g(Y, Z) h X-2 g(X, h Y) Z \\
-2 g(h Y, Z) X+4 g(h X, Z) Y+4 g(X, Z) h Y+2 g(h X, Z) h Y \\
+g(\varphi h Y, X) \varphi h Z+g(\varphi h Y, Z) \varphi h X-2 g(\varphi h X, Z) \varphi h Y\} .
\end{gathered}
$$

Adding (4.13) and (4.14) and using Bianchi's first identity, $\varphi$ is antisymmetric and $\varphi h$ is symmetric we get

$$
\begin{gathered}
4 R(X, Y) Z=(H(P)+3)\{g(Y, Z) X-g(X, Z) Y\} \\
+(H(P)-1)\{2 g(X, \varphi Y) \varphi Z+g(X, \varphi Z) \varphi Y-g(Y, \varphi Z) \varphi X\} \\
-2\{g(h X, Z) h Y+2 g(X, Z) h Y+2 g(h X, Z) Y-2 g(h Y, Z) X \\
-2 g(Y, Z) h X-g(h Y, Z) h X-g(\varphi h X, Z) \varphi h Y+g(\varphi h Y, Z) \varphi h X\}
\end{gathered}
$$

for any $X, Y, Z$ orthogonal to $\xi$. Moreover, using (3.1) and $h \xi=\varphi \xi=0$, we conclude that (4.15) is valid for any $Z$ and for $X, Y$ orthogonal to $\xi$. Now, let $X, Y, Z$ be arbitrary vectors of $T_{P} M$. Writing

$$
X=X_{T}+\eta(X) \xi, \quad Y=Y_{T}+\eta(Y) \xi
$$

where $g\left(X_{T}, \xi\right)=g\left(Y_{T}, \xi\right)=0$, and using (3.1), (3.3) and $h \xi=0$, then (4.15) gives (4.1) after a straightforward calculation.

Now, we will prove that the $\varphi$-sectional curvature is constant. Let $\left\{X_{i}\right\}, i=$ $1, \cdots, 2 n+1$, be a local orthonormal frame. Putting $Y=Z=X_{i}$ in (4.1), adding with respect to $i$ and using (2.1)-(2.4) we get the following formula, for the Ricci operator, at any point of $M$ :

$$
\begin{gathered}
2 Q=\{(n+1) H+3(n-1)+2 \kappa\} I-\{(n+1) H+3(n-1) \\
-2 \kappa(2 n-1)\} \eta \otimes \xi+2\{2(n-1)+\mu\} h .
\end{gathered}
$$


Comparing this with (3.6), which is valid on any $(\kappa, \mu)$-contact Riemannian manifold with $\kappa \neq 1$, we get $(n+1) H=n-1-2 n \mu-2 \kappa$, i.e. $H$ is constant. On the other hand, from (3.5) we have $H=-\kappa-\mu$. Comparing the two last equations we get $(n-1)(\mu-\kappa-1)=0$. Moreover, since $n>1$, we have $\mu=\kappa+1$ and so $H=-2 \kappa-1$. This completes the proof of the theorem.

In Theorem 4.1 we proved that $\mu=\kappa+1$, in the case where the non Sasakian, $(\kappa, \mu)$-contact Riemannian manifold has constant $\varphi$-sectional curvature. Now we will prove the inverse, i.e. supposing $M(\eta, \xi, \varphi, g)$ is a $(2 n+1)$-dimensional $(n>1)$, non Sasakian, $(\kappa, \mu)$-contact Riemannian manifold with

$$
\mu=\kappa+1
$$

we will prove that $M$ has constant $\varphi$-sectional curvature.

Let $X \in T_{P} M$ be a unit vector orthogonal to $\xi$. By Lemma 3.1 we can write

$$
X=X_{\lambda}+X_{-\lambda} \quad \text { where } X_{\lambda} \in D(\lambda) \text { and } X_{-\lambda} \in D(-\lambda) \text {. }
$$

Using Lemma 3.1, Theorem 3.2, (2.3), we get, after a long straightforward calculation,

$$
K(X, \varphi X)=-(\kappa+\mu)+4(\kappa-\mu+1)\left(g\left(X_{\lambda}, X_{\lambda}\right) g\left(X_{-\lambda}, X_{-\lambda}\right)-g\left(X_{\lambda}, \varphi X_{-\lambda}\right)^{2}\right)
$$

and hence by $(4.16), K(X, \varphi X)=-(\kappa+\mu)=$ const. So we have proved the following theorem.

THEOREM 4.2. Let $M(\eta, \xi, \varphi, g)$ be a $(2 n+1)$-dimensional $(n>1)$, non Sasakian, $(\kappa, \mu)$-contact Riemannian manifold. Then $M$ has constant $\varphi$-sectional curvature if and only if $\mu=\kappa+1$.

An immediate consequence of Theorems 4.2 and 3.4 is the following theorem.

THEOREM 4.3. Let $M$ be an n-dimensional Riemannian manifold, $n>2$, of constant sectional curvature $c$. The tangent sphere bundle $T_{1} M$ has constant $\varphi$-sectional curvature $\left(c^{2}\right)$ if and only if $c=2 \pm \sqrt{5}$.

REMARK. The tangent sphere bundle $T_{1} M$, of a 2-dimensional Riemannian manifold $M$ of constant sectional curvature $c$, has constant $\varphi$-sectional curvature $c^{2}$ for any $c \neq 1$, as follows from (3.5) and Theorem 3.4.

\section{Examples.}

1. The first non-trivial example of a 3-dimensional, non Sasakian, contact Riemannian manifold of constant $\varphi$-sectional curvature was given in [5]. In [3] there exist more examples concerning the 3 -dimensional case.

2. Theorem 4.3 gives two examples of $(2 n+1)$-dimensional, $n>1$, non Sasakian, $(\kappa, \mu)$-contact Riemannian manifolds of positive constant $\varphi$-sectional curvature, equal to $(2 \pm \sqrt{5})^{2}$. 
3. Now, we will give a method to construct non Sasakian, $(\kappa, \mu)$-contact Riemannian manifolds of constant $\varphi$-sectional curvature. Let $M(\eta, \xi, \varphi, g)$ be a $(2 n+1)$ dimensional, $(\kappa, \mu)$-contact Riemannian manifold $(n>1, \kappa \neq 1, \mu<2)$. The existence of such a manifold follows from Theorem 3.4, taking $c>-1(c \neq 1)$. By a $D$-homothetic deformation [7] we mean a change of structure tensors of the form

$$
\bar{\eta}=a \eta, \quad \bar{\xi}=\frac{1}{a} \xi, \quad \bar{\varphi}=\varphi, \quad \bar{g}=a g+a(a-1) \eta \otimes \eta
$$

where $a$ is a positive constant. It is well known [3] that $M(\bar{\eta}, \bar{\xi}, \bar{\varphi}, \bar{g})$ is a new $(\bar{\kappa}, \bar{\mu})$ contact Riemannian manifold with

$$
\bar{\kappa}=\frac{\kappa+a^{2}-1}{a^{2}} \text { and } \bar{\mu}=\frac{\mu+2 a-2}{a} .
$$

Choosing $a=(\kappa-1) /(\mu-2)>0, M(\bar{\eta}, \bar{\xi}, \bar{\varphi}, \bar{g})$ has constant $\varphi$-sectional curvature. In fact, substituting $a$ in (5.1) we get $\bar{\mu}=\bar{\kappa}+1$ and so by Theorem $4.2, M(\bar{\eta}, \bar{\xi}, \bar{\varphi}, \bar{g})$ has constant $\varphi$-sectional curvature

$$
\bar{H}=-\bar{\kappa}-\bar{\mu}=1-2 \bar{\mu}=\left(2(\mu-2)^{2}-3(1-\kappa)\right) /(1-\kappa) .
$$

So $\bar{H}$ is positive, negative or zero if

$$
A>0, A<0 \text {, or } A=0, \quad \text { where } A=2(\mu-2)^{2}-3(1-\kappa), \quad(\mu<2)
$$

respectively. The existence of a $(\kappa, \mu)$-contact Riemannian manifold satisfying $A>0$ or $A<0$ or $A=0$ follows easily from Theorem 3.4 .

The above examples give an answer (for $n>1$ ) to the following remark of Tanno (see [8], p. 445). "It seems to be an open problem if there exist contact Riemannian manifolds of constant $\varphi$-sectional curvature, which are not Sasakian".

\section{References}

[ 1 ] D. E. BlaIR, Contact Manifolds in Riemannian Geometry, Lecture Notes in Math., 509 (1976), Springer.

[2] D. E. BlaIR, Two remarks on contact metric structures, Tôhoku Math. J. 29 (1977), 319-324.

[3] D. E. Blair, T. Koufogiorgos and P. J. Papantoniou, Contact metric manifolds satisfying a nullity condition, Israel J. of Math. 91 (1995), 189-214.

[4] H. ENDO, On the curvature tensor fields of a type of contact metric manifolds and of its certain submanifolds, submitted.

[ 5] T. Koufogiongos, Contact metric manifolds, Ann. Global Anal. Geom. 11 (1993), 25-34.

[6] K. OGIUE, On almost contact manifolds admiting axiom of planes or axiom of free mobility, Kodai Math. Sem. Rep. 16 (1964), 223-232.

[ 7 ] S. TANNo, The topology of contact Riemannian manifolds, Illinois J. Math. 12 (1968), 700-717.

[8] S. TANNO, Ricci curvatures of contact Riemannian manifolds, Tôhoku Math. J. 40 (1988), 441-448.

[ 9 ] Y. TASHIRO, On contact structure of tangent sphere bundles, Tôhoku Math. J. 21 (1969), 117-143. 
Present Address:

DePartment of MATHEMATICS, University of IoANNina,

IOANNINA 451 10, GREECE.

e-mail: tkoufog@cc.uoi.gr 